\title{
Rationale, Design, and Baseline Characteristics of the Prospective Japan Acute Myocardial Infarction Registry (JAMIR)
}

\author{
Satoshi Honda ${ }^{1}$ Kensaku Nishihira ${ }^{1}$. Sunao Kojima ${ }^{2}$ - Misa Takegami ${ }^{3}$. Yasuhide Asaumi ${ }^{1}$. Makoto Suzuki ${ }^{4}$. \\ Masami Kosuge ${ }^{5}$. Jun Takahashi ${ }^{6} \cdot$ Yasuhiko Sakata $^{6} \cdot$ Morimasa Takayama $^{4} \cdot$ Tetsuya Sumiyoshi $^{4} \cdot$ Hisao Ogawa $^{1}$. \\ Kazuo Kimura ${ }^{5}$. Satoshi Yasuda ${ }^{1} \cdot$ on behalf of the JAMIR investigators
}

Published online: 23 November 2018

(C) The Author(s) 2018

\begin{abstract}
Background Antiplatelet therapy is a cornerstone of treatment following acute myocardial infarction (AMI). Recently, prasugrel, a new and potent antiplatelet agent, has been introduced in clinical practice. To date, however, real-world in-hospital and followup data in Japanese patients with AMI remain limited.

Objectives To examine ischemic and bleeding events in Japanese patients with AMI and the association between these events and antiplatelet therapy.

Methods The Japan AMI Registry (JAMIR) is a multicenter, nationwide, prospective registry enrolling patients with AMI from 50 institutions. The inclusion criterion is spontaneous onset of AMI diagnosed based on either the universal definition or Monitoring Trends and Determinants in Cardiovascular disease (MONICA) criteria. The major exclusion criteria are hospital admission $\geq 24 \mathrm{~h}$ after onset, no return of spontaneous circulation on admission following out-of-hospital cardiopulmonary arrest, and AMI as a complication of percutaneous coronary intervention or coronary artery bypass grafting. The primary end point of the study is the composite of cardiovascular death, non-fatal myocardial infarction, and non-fatal stroke. Major safety end points include major bleeding based on Thrombolysis in Myocardial Infarction (TIMI) criteria and type 3 or type 5 bleeding based on Bleeding Academic Research Consortium (BARC) criteria. Between December 2015 and May 2017, a total of 3411 patients (mean age $68.1 \pm 13.2$ years, $23.4 \%$ female) were enrolled in the study. Patients will be followed for 1 year.

Conclusions JAMIR will provide important information regarding contemporary practice patterns in the management of Japanese patients with AMI, their demographic and clinical characteristics, in-hospital and post-discharge outcomes, and how they are related to antiplatelet therapy.
\end{abstract}

Keywords Acute myocardial infarction $\cdot$ Bleeding event $\cdot$ Antiplatelet therapy

Despite major advances in prevention and treatment, cardiovascular disease is the second leading cause of mortality in Japan. It continues to be a major public health burden, and its prevalence is expected to increase with an increasing aging society and lifestyle changes [1,2]. Among cardiovascular diseases, acute myocardial infarction (AMI) is a major cause of mortality and disability.

Electronic supplementary material The online version of this article (https://doi.org/10.1007/s10557-018-6839-1) contains supplementary material, which is available to authorized users.

Satoshi Yasuda

yasuda.satoshi.hp@ncvc.go.jp

1 Department of Cardiovascular Medicine, National Cerebral and Cardiovascular Center, 5-7-1 Fujishiro-dai, Osaka, Suita 565-8565, Japan

2 Department of General Internal Medicine 3, Kawasaki Medical School, Okayama, Japan
3 Department of Preventive Medicine and Epidemiologic Informatics, National Cerebral and Cardiovascular Center, Osaka, Japan

4 Department of Cardiology, Sakakibara Heart Institute, Fuchu, Tokyo, Japan

5 Department of Cardiovascular Medicine, Yokohama City University Medical Center, Yokohama, Japan

6 Department of Cardiovascular Medicine, Tohoku University, Sendai, Japan 
In the past few decades, various types of studies on AMI have been carried out to improve treatment [3, 4]. Most of the evidence has been provided by studies carried out in Western countries [4]. However, Japanese individuals have different physiques, genetic backgrounds, and medical environments [5]. It is unknown whether evidence from Western countries could be generalized to clinical practice in Japan. Consequently, the Japan Acute Myocardial Infarction Registry (JAMIR) was initiated to generate original data about Japanese subjects. Recently, a retrospective analysis was performed [6].

Antiplatelet therapy is a cornerstone of treatment to prevent thrombotic complications after AMI [7, 8]. Dual antiplatelet therapy (DAPT) with aspirin and clopidogrel is the conventional gold standard for antiplatelet therapy after AMI and coronary stenting. However, clopidogrel exhibits significant interpatient variability in antiplatelet response, mainly because of genetic variations in cytochrome P450 (CYP) 2C19 [9]. Of note, decreased response to clopidogrel is common among Asians due to a high prevalence of genetic polymorphisms [10-12]. Indeed, the prevalence of CYP2 C19*3 allelic variants, a major loss-of-function polymorphism in CYP2C19, is $24.2 \%$ among Japanese, $14.8 \%$ among Koreans, but only $0.4 \%$ among Africans and $0.2 \%$ among Caucasians [11].

Recently, prasugrel has been introduced as a new potent antiplatelet agent. Prasugrel is expected to be a better antiplatelet agent than clopidogrel because it exerts its antiplatelet effect more rapidly, and there are fewer individual differences in metabolism $[13,14]$. In the TRITON-TIMI 38 trial, the safety and efficacy of prasugrel and clopidogrel were compared in patients with acute coronary syndrome (ACS) [15]. At a median follow-up of 14.5 months, the incidence of ischemic events with a 60-mg loading dose and a 10-mg maintenance dose of prasugrel was $19 \%$ lower than with clopidogrel (prasugrel arm, 9.9\% vs. clopidogrel arm, 12.1\%). Importantly, subanalyses of TRITON-TIMI 38 trial demonstrated that common functional CYP genetic variants were associated with diminished platelet inhibition and higher rates of cardiovascular events in patients treated with clopidogrel but not in those treated with prasugrel $[16,17]$. However, the incidence of bleeding events was $32 \%$ higher in the prasugrel group (prasugrel arm, $2.4 \%$ vs. clopidogrel arm, 1.8\%). Since the TRITON-TIMI 38 trial was conducted in 30 Western and South American countries, but no Asian countries, it is unclear whether these results can be simply extrapolated to Asian populations. Asian populations have a lower risk of ischemic events and a higher risk of bleeding events than Western populations [18-20]. Therefore, it is possible that a lower level of platelet inhibition would be sufficient in Asian populations than in Western populations. In this context, the PRASFITACS study was carried out to establish the appropriate dosage of prasugrel for Japanese patients with ACS. In the trial, a 20mg loading dose and a 3.75-mg maintenance dose of prasugrel were compared with conventional doses of clopidogrel. At
24 weeks of follow-up, the incidence of major adverse cardiovascular events was numerically lower in the prasugrel group than in the clopidogrel group (9.4\% vs. $11.8 \%)$. There was no apparent increase in the incidence of bleeding events $(1.9 \%$ vs. $2.2 \%$ ). Based on these results, a loading dose of $20 \mathrm{mg}$ and a maintenance dose of $3.75 \mathrm{mg}$ were established for prasugrel in Japan. Prasugrel was approved in 2014 in Japan. It has been used in the treatment of patients with ACS [21]. However, real-world clinical data on Japanese patients with AMI remain limited. Therefore, the present JAMIR study was designed to prospectively assess ischemic and bleeding events in Japanese patients with AMI and their association with antiplatelet therapy.

\section{Methods}

\section{Study Population}

We registered all consecutive patients presenting with spontaneous onset of AMI between December 2015 and May 2017 at 50 institutions (Fig. 1). Table 1 summarizes the registry's inclusion and exclusion criteria. AMI was diagnosed based on either the MONICA criteria or the universal definition $[22,23]$. We excluded patients who were admitted to the hospital $\geq 24 \mathrm{~h}$ after onset, with no return of spontaneous circulation on admission after out-of-hospital cardiopulmonary arrest, or had AMI as a complication of percutaneous coronary intervention (PCI) or coronary artery bypass grafting (CABG). Management of patient was dependent on the discretion of treating physician, and antiplatelet regimen and dosage were determined according to the guidelines of the Japanese Circulation Society [24, 25].

\section{Study End Points}

The primary end point of the study is the composite of cardiovascular death, non-fatal myocardial infarction, and non-fatal stroke. Cardiovascular death is defined as any death with a demonstrable cardiovascular cause or any death that is not clearly attributable to a non-cardiovascular cause. Myocardial infarction must be distinct from the index event and is defined based on symptoms suggestive of ischemia or infarction, electrocardiographic data, cardiac biomarker data, or pathological evidence of infarction depending on the clinical situation based on criteria adapted from the American College of Cardiology definitions (Bethesda, MD) [26, 27]. Major safety end points include major bleeding based on the Thrombolysis in Myocardial Infarction (TIMI) criteria and type 3 or 5 bleeding based on the Bleeding Academic Research Consortium (BARC) criteria [28, 29].

Secondary end points include a composite of ischemic events (cardiovascular death, non-fatal myocardial infarction, 
Fig. 1 Number of participating institutions by location (prefecture)

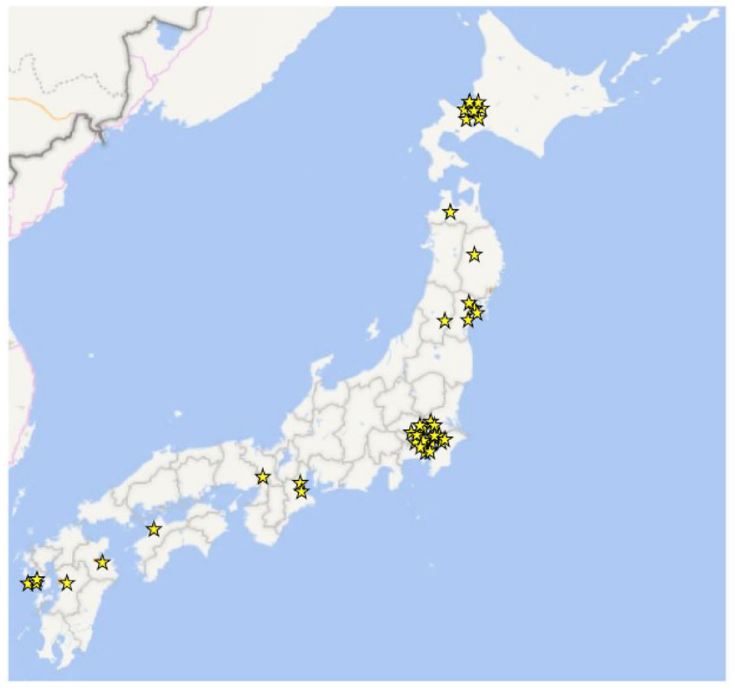

\begin{tabular}{lr} 
Hokkaido & 7 \\
Aomori & 1 \\
Miyagi & 4 \\
Yamagata & 1 \\
Iwate & 1 \\
Tokyo & 22 \\
Kanagawa & 5 \\
Mie & 2 \\
Osaka & 1 \\
Ehime & 1 \\
Oita & 1 \\
Kumamoto & 1 \\
Nagasaki & 3 \\
\hline Total & 50
\end{tabular}

and non-fatal cerebral infarction) and bleeding events (major bleeding based on TIMI criteria); individual components of ischemic events; all-cause death; stent thrombosis; major and minor bleeding based on TIMI criteria; type 2, 3, or type 5 bleeding based on the BARC criteria; and hospital readmission due to heart failure. Stent thrombosis was defined as definite or probable stent thrombosis according to the Academic Research Consortium definition [30].

\section{Data Collection}

All patients who met the eligibility criteria were registered at each study site. Primary data collection is derived from the patient's medical record. Information was collected on patient demographics, medical history, ambulance use, details about coronary angiography and invasive therapy, cardiac medications, and outcomes (Table 2). Investigators, clinical research coordinators, or local data managers at each study site registered the data using the JAMIR registration system. The data were anonymized in a linkable manner at each study site before they were sent. A follow-up study of patients was conducted 1 year after the onset of AMI based on the medical information available at each study site. A letter requesting for

Table 1 Inclusion and exclusion criteria

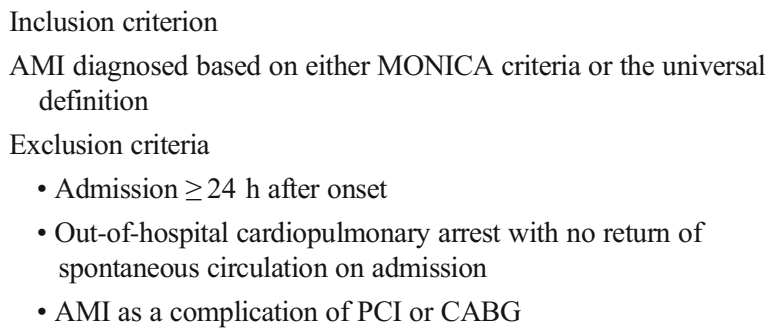

- Out-of-hospital cardiopulmonary arrest with no return of spontaneous circulation on admission

- AMI as a complication of PCI or CABG

$A M I$ acute myocardial infarction, $P C I$ percutaneous coronary intervention, $C A B G$ coronary artery bypass grafting follow-up was sent to patients whose medical information was not available at the study sites after 1 year due to hospital transfer or other reasons. When the letter was sent, appropriate informed consent was obtained.

\section{Data Analysis}

Univariate and multivariate analyses will be performed to identify determinants of ischemic and bleeding events after AMI. All pre-specified and exploratory analyses will be performed by the JAMIR group using SAS (SAS Institute, Inc., Cary, NC, USA).

Baseline continuous variables will be presented as means \pm SD or medians and interquartile range, depending on the distribution of the data. Categorical variables will be presented as counts and percentages.

\section{Study Organization}

This prospective study is planned by the Japan Cardiovascular Research Foundation and is coordinated by the JAMIR group. IQVIA Services Japan serves as the contract research organization and provides data and site management services. The steering committee is responsible for the scientific content of the protocol, protocol implementation, results presentation, and manuscript preparation. Trial operations are monitored and coordinated by the operation committee.

\section{Baseline Patient Characteristics}

JAMIR started enrollment in December 2015. By the end of July 2017, a total of 3411 patients were registered from 50 sites across Japan (Fig. 2). All institutes except one used the universal definition for the diagnosis of AMI, and $99 \%$ of study patients (3371 of 3411) were diagnosed by the universal 
Table 2 Variables in JAMIR

Clinical demographics

Invasive procedures

Test results during hospitalization

Medications during hospitalization

Complications during hospitalization

Medications after discharge

Test results during follow-up

Outcomes
Age, sex, height, weight, ambulance use, presence of out-of-hospital cardiac arrest, date of admission, STEMI or NSTEMI, Killip class, systolic and diastolic blood pressure on admission, heart rate on admission, presence of hypertension, presence of diabetes, presence of dyslipidemia, smoking, previous myocardial infarction, history of CABG, history of PCI, history of atrial fibrillation, history of cerebrovascular disease, history of malignancy, history of peripheral artery disease

Use of emergency CAG, approach site, culprit lesion site, number of diseased vessels, use of reperfusion therapy

(PCI or thrombolytic therapy), use of stenting, stent type (drug-eluting stent or bare metal stent), date of reperfusion, door-to-device time, TIMI classification on final angiography, concurrent PCI in non-culprit lesion, use of IABP, use of V-A ECMO, use of CABG

Peak serum CK, peak serum CK-MB, peak troponin T, serum creatinine, hemoglobin on admission, blood glucose on admission, total cholesterol, LDL cholesterol, HDL cholesterol, triglycerides, LVEF on echocardiography

Antiplatelet agents (loading and maintenance doses), ACE inhibitors, ARBs, $\beta$-blockers, statins, anticoagulants (warfarin or DOAC), proton pump inhibitor

Cardiogenic shock, cardiac rupture, ventricular septal perforation, acute mitral regurgitation (papillary muscle rupture), right ventricular involvement

Antiplatelet agents, statins, anticoagulants

Total cholesterol, LDL cholesterol, HDL cholesterol, triglycerides

Cardiovascular death, non-cardiovascular death, non-fatal myocardial infarction, non-fatal cerebral infarction, major and minor bleeding based on TIMI criteria, type 2, 3, or type 5 bleeding based on BARC criteria, stent thrombosis, readmission due to heart failure

JAMIR Japan Acute Myocardial Infarction Registry, STEMI ST elevation myocardial infarction, NSTEMI non-ST elevation myocardial infarction, $C A B G$ coronary artery bypass grafting, $P C I$ percutaneous coronary intervention, $C A G$ coronary angiography, IABP intra-aortic balloon pumping, $V-A E C M O$ venoarterial extracorporeal membrane oxygenation, $C K$ creatine kinase, $L D L$ cholesterol low-density lipoprotein cholesterol, $H D L$ cholesterol high-density lipoprotein cholesterol, $L V E F$ left ventricular ejection fraction, $A C E$ angiotensin-converting enzyme, $A R B$ angiotensin receptor blocker, $D O A C$ direct oral anticoagulant, TIMI Thrombolysis in Myocardial Infarction, $B A R C$ Bleeding Academic Research Consortium

Fig. 2 Number of patients enrolled in Japan Acute Myocardial Infarction Registry

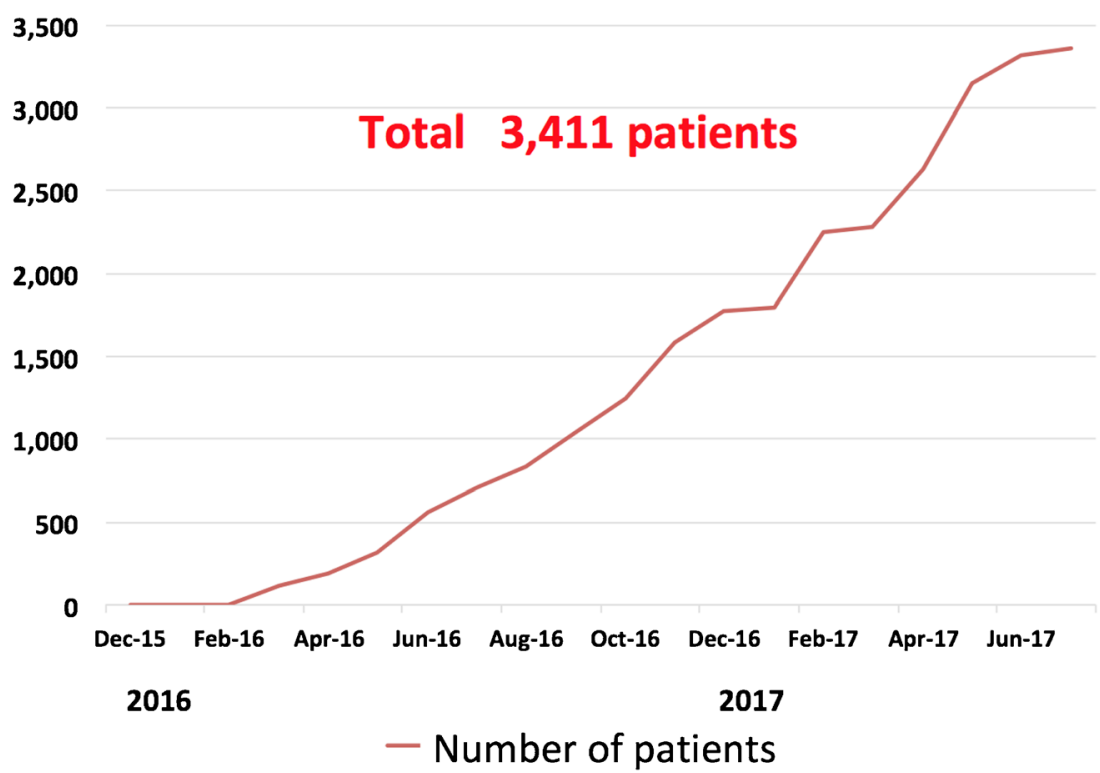


definition. Patient characteristics are presented in Table 3. The mean age was $68.1 \pm 13.2$ years, and $23.4 \%$ was female. ST elevation myocardial infarction (STEMI) accounted for $77 \%$ of patients with AMI. With respect to infarct location, anterior was the most frequent $(51 \%)$, followed by inferior $(41 \%)$, and lateral or posterior $(10 \%)$. The prevalence of coronary risk factors was $75.1 \%$ for hypertension, $38.7 \%$ for diabetes, and $71.7 \%$ for dyslipidemia. Ten percent of patients had a prior myocardial infarction, $8.8 \%$ of patients had a history of atrial fibrillation, and $9.9 \%$ of patients had malignancy. Of note, $97 \%$ of patients underwent emergent coronary angiography and primary PCI defined as emergent or urgent PCI was performed in $93 \%$ of patients overall. During hospitalization, almost all patients were treated with aspirin (97.2\%). The most frequent P2Y12 inhibitor used was prasugrel (80.6\%), followed by clopidogrel (17.2\%). Warfarin and direct oral anticoagulants (DOACs) were administered to $6.2 \%$ and $7.4 \%$ of patients, respectively. With respect to the dose of prasugrel, $96.2 \%$ of patients received $3.75 \mathrm{mg}$ (Table 4 ).

Table 3 Baseline characteristics $(n=3411)$

\begin{tabular}{ll}
\hline Age (years) & $68.1 \pm 13.2$ \\
Gender, female (\%) & 23.4 \\
STEMI (\%) & 77.0 \\
Killip class $\geq$ II (\%) & 23.3 \\
Hypertension (\%) & 74.4 \\
Diabetes (\%) & 36.2 \\
Dyslipidemia (\%) & 70.7 \\
Prior stroke (\%) & 9.9 \\
Prior myocardial infarction (\%) & 9.7 \\
PAD (\%) & 4.3 \\
Prior CABG (\%) & 2.5 \\
Prior PCI (\%) & 11.6 \\
Atrial fibrillation (\%) & 6.9 \\
Malignancy (\%) & 8.7 \\
Current smoking (\%) & 41.9 \\
Medication during hospitalization (\%) & \\
$\quad$ Antiplatelet therapy & \\
$\quad$ Aspirin & \\
$\quad$ Prasugrel & 97.2 \\
$\quad$ Clopidogrel & 80.6 \\
Ticlopidine & 17.2 \\
Warfarin & 0.3 \\
DOAC & 6.2 \\
Emergent coronary angiography (\%) & 7.4 \\
Thrimary PCI (\%) & 97.0 \\
\hline
\end{tabular}

Primary PCI is defined as the emergent or urgent use (within $24 \mathrm{~h}$ after admission) of PCI

STEMI ST elevation myocardial infarction, $P A D$ peripheral artery disease, $C A B G$ coronary artery bypass grafting, $P C I$ percutaneous coronary intervention, $D O A C$ direct oral anticoagulant
Table 4 Maintenance doses of antiplatelet drugs

\begin{tabular}{lll}
\hline & Maintenance dose & Percent \\
\hline Aspirin & $100 \mathrm{mg}$ & 95.5 \\
& $81 \mathrm{mg}$ & 3.2 \\
& Other & 1.3 \\
Prasugrel & $3.75 \mathrm{mg}$ & 96.2 \\
& Other & 3.8 \\
Clopidogrel & $75 \mathrm{mg}$ & 95.1 \\
& Other & 4.9 \\
\hline
\end{tabular}

\section{Discussion}

JAMIR is a registry of Japanese patients with AMI designed to be a multicenter, nationwide, prospective observational study with the primary objective of examining ischemic and bleeding events and their relationship with antiplatelet therapy. A total of 3411 patients were enrolled between December 2015 and May 2017; soon after prasugrel, a potent antiplatelet agent, was introduced in Japan.

The Prevention of Atherothrombotic Incidents Following Ischemic Coronary Attack (PACIFIC) registry is a multicenter, prospective, observational study of Japanese patients with ACS [31]. Between May 2008 and May 2009, 3597 consecutive patients aged $\geq 20$ years hospitalized for ACS were enrolled at 96 hospitals and followed for 2 years. The majority (93.5\%) of patients underwent PCI, with a success rate of 93.9\% . Antiplatelet agents were prescribed to $99.3 \%$ of patients, and $92.6 \%$ of the entire group received DAPT (e.g., aspirin plus clopidogrel). The cumulative incidence of major adverse cardiac and cerebrovascular events (MACCEs) was $6.4 \%$, and all-cause mortality was $6.3 \%$. However, due to pharmacological limitations of clopidogrel (interindividual variability in biological efficacy, slow onset of action, mild inhibition of platelet reactivity), ischemic recurrences remained common following stent implantation, especially in patients with ACS. Thus, more potent P2Y12 inhibitors, such as prasugrel and ticagrelor, were developed to overcome these pitfalls. New P2Y12 inhibitors have a considerable safety and tolerability profile and are associated with lower mortality in patients undergoing PCI compared with clopidogrel; however, the risk/benefit ratio of ischemic and bleeding events should be further investigated in real-world clinical practice.

Indeed, the recent Korean AMI Registry (KAMIR) study demonstrated that prasugrel and ticagrelor had similar rates of MACCEs at 1 year, but a higher rate of bleeding events compared with clopidogrel (MACCEs, clopidogrel $2.1 \%$ vs. prasugrel $2.6 \%$ vs. ticagrelor $2.1 \%, p=0.790$; bleeding events, clopidogrel $3.1 \%$ vs. prasugrel $8.0 \%$ vs. ticagrelor $8.0 \%, p<0.001$ ) [32]. However, it should be noted that Korean patients who underwent PCI received $300 \mathrm{mg}$ aspirin and $60 \mathrm{mg}$ prasugrel or $180 \mathrm{mg}$ ticagrelor as a loading dose 
prior to PCI. The maintenance dose after PCI was 100 $300 \mathrm{mg}$ aspirin and/or 5 or $10 \mathrm{mg}$ prasugrel once daily or $90 \mathrm{mg}$ ticagrelor twice daily [33]. Despite having an East Asian study population, this study included doses of prasugrel that are different from that of Japan; thus, further studies like the present JAMIR study are warranted to adapt Western guidelines on new potent P2Y12 inhibitors for East Asians.

With regard to the definition of AMI, MONICA criteria had been widely used in Japan [23]. The first universal definition was developed in 2000 [34], and the third universal definition was published in 2012 [22]. Although the universal definition based on high-sensitive troponin measurement was becoming common in Japan, we did not know how much participating hospital applied the universal definition when we planned the JAMIR project. Accordingly, we allowed both definitions for the JAMIR; however, as a result, all institutes except for one used the third universal definitions. This result suggests that diagnosis of AMI based on high-sensitive troponin measurement has become common in Japan.

\section{Conclusion}

This study will provide important information regarding contemporary practice patterns in the management of Japanese patients with AMI, their demographic and clinical characteristics, and in-hospital and post-discharge outcomes.

Acknowledgments We thank all the investigators, clinical research coordinators, and data managers involved in the JAMIR study for their contributions. The JAMIR study group consists of the following institutions and members:

Hokkaido Medical Center: Takashi Takenaka; Hirosaki University: Hirofumi Tomita, Hiroaki Yokoyama; Iwate Medical University: Tomonori Ito, Masaru Ishida, Yorihiko Koeda; Yamagata University: Masafumi Watanabe, Tetsu Watanabe, Taku Toshima; Tohoku University: Hiroaki Shimokawa, Yasuhiko Sakata, Jun Takahashi, Kiyotaka Hao; Sakakibara Heart Institute: Tetsuya Sumiyoshi, Morimasa Takayama, Makoto Suzuki; Yokohama City University Hospital: Kazuo Kimura, Masami Kosuge, Toshiaki Ebina; Showa University Fujigaoka Hospital: Hiroshi Suzuki, Atsuo Maeda; Mie University Hospital: Masaaki Ito, Tairo Kurita, Jun Masuda; Matsuzaka Central General Hospital: Takashi Tanigawa; Ehime University Hospital: Jitsuo Higaki, Kazuhisa Nishimura; Oita University: Naohiko Takahashi, Hidefumi Akioka, Kyoko Kawano; Nagasaki University: Koji Maemura, Yuji Koide; Kumamoto University: Sunao Kojima, Kenichi Tsujita; National Cerebral And Cardiovascular Center: Hisao Ogawa, Satoshi Yasuda, Yasuhide Asaumi, Kensaku Nishihira, Yoshihiro Miyamoto, Misa Takegami, Yoko Nakao, Satoshi Honda; Nagasaki Harbor Medical Center: Hiroshi Nakajima; Isahaya General Hospital: Kenji Yamaguchi; Sapporo City General Hospital: Takao Makino; Sapporo Cardiovascular Clinic: Daitarou Kanno; Teine Keijinkai Hospital: Yasuhiro Omoto; Hokkaido Cardiology Hospital: Daisuke Hotta; Sapporo Kosei General Hospital: Toshiya Sato; Nippon Medical School Musashi Kosugi Hospital: Naoki Sato, Arifumi Kikuchi, Michiko Sone, Koji Takagi; Ayase Heart Hospital: Imun Tei; Tokyo Metropolitan Hiroo General Hospital: Takashi Shibui, Sho Nagamine; Nippon Medical School Hospital: Wataru Shimuzu, Takeshi Yamamoto; Tokyo Saiseikai Central Hospital: Toshiyuki Takahashi; Tokyo Medical Center: Yukihiko
Momiyama; St. Luke's International Hospital: Atsushi Mizuno; Edogawa Hospital: Hiroshi Ohira; Kyorin University Hospital: Hideaki Yoshino, Youhei Shigeta; Nihon University Itabashi Hospital: Atsushi Hirayama, Yasuo Okurmura, Daisuke Fukamachi, Tadateru Takayama; Toho University Omori Medical Center: Hiroki Niikura, Hiroki Takenaka; Mitsui Memorial Hospital: Shuzo Tanimoto, Kazuyuki Yahagi; Tokyo Metropolitan Tama Medical Center: Hiroyuki Tanaka; Disaster Medical Center: Yasuhiro Sato, Ohno Masakazu; Musashino Red Cross Hospital: Takamichi Miyamoto, Nobuhiro Hara; NTT Medical Center: Mikio Kishi; Oume Municipal General Hospital: Sigeo Shimizu, Ken Kurihara; Oghikubo Hospital: Yasuhiro Ishii; Teikyo University Hospital: Ken Kozuma, Yusuke Watanabe; Fraternity Memorial Hospital: Yasuhiro Takahashi; Jikei University Hospital: Michihiro Yoshimura, Satoshi Morimoto; Tokyo Women's Medical University Hospital: Nobuhisa Hagiwara, Yuichiro Minami; Tokyo Medical University Hospital: Jun Yamashita; Osaki Citizen Hospital: Kaoru Iwabuchi, Takeshi Yamauchi; Sendai Open Hospital: Atsushi Kato, Shigeto Namiuchi; Sendai Mecical Center: Tsuyoshi Shinozaki; and Kesennuma City Hospital: Kazunori Ogata, Ryuji Tsuburaya.

Source of Funding This work was planned by the Japan Cardiovascular Research Foundation and is financially supported by Dai-ichi Sankyo, Ltd.

\section{Compliance with Ethical Standards}

Conflict of Interest Dr. Yasuda reports remuneration for lectures from Takeda, Daiichi Sankyo, and Bristol-Myers Squibb and trust research/ joint research funds from Takeda and Daiichi Sankyo; Dr. Takayama reports lecture fees from Daiichi Sankyo; and Dr. Ogawa reports lecture fees and research grants from AstraZeneca, Bayer, Boehringer Ingelheim,Daiichi Sankyo, Pfizer, and Sanofi. Other authors have no conflict of interest.

Ethical Approval This study has been conducted in accordance with the ethical guidelines for medical research on humans laid out in the Declaration of Helsinki. This research protocol was designed by the authors and approved by the Institutional Review Board of the National Cerebral and Cardiovascular Center and local ethics committees or local institutional review board at each study site. Although informed consent was not obtained due to the observational nature of this registry, details about the study were posted on a website and at the study sites (opt-out) to inform the subjects of the content and timeline of the study and to ensure that they have the opportunity to refuse participation in this registry. In addition, the research secretariat confirmed compliance with opt-out procedures at each study site. This study is registered with the Japanese UMIN Clinical Trials Registry (UMIN000019479).

Open Access This article is distributed under the terms of the Creative Commons Attribution 4.0 International License (http:// creativecommons.org/licenses/by/4.0/), which permits unrestricted use, distribution, and reproduction in any medium, provided you give appropriate credit to the original author(s) and the source, provide a link to the Creative Commons license, and indicate if changes were made.

\section{References}

1. Iso H. Changes in coronary heart disease risk among Japanese. Circulation. 2008;118(25):2725-9.

2. Ohira T, Iso H. Cardiovascular disease epidemiology in Asia: an overview. Circ J. 2013;77(7):1646-52.

3. Ibanez B, James S, Agewall S, Antunes MJ, Bucciarelli-Ducci C, Bueno H, et al. 2017 ESC guidelines for the management of acute 
myocardial infarction in patients presenting with ST-segment elevation: the task force for the management of acute myocardial infarction in patients presenting with ST-segment elevation of the European Society of Cardiology (ESC). Eur Heart J. 2018;39(2): 119-77.

4. Group JCSJW. Guidelines for secondary prevention of myocardial infarction (JCS 2011). Circ J. 2013;77(1):231-48.

5. Iso H. Lifestyle and cardiovascular disease in Japan. J Atheroscler Thromb. 2011;18(2):83-8.

6. Kojima S, Nishihira K, Takegami M, Nakao YM, Honda S, Takahashi J, et al. Nationwide real-world database of 20,462 patients enrolled in the Japanese Acute Myocardial Infarction Registry (JAMIR): impact of emergency coronary intervention in a super-aging population. Int J Cardiol Heart Vasc. 2018;20:1-6.

7. Selwyn AP. Prothrombotic and antithrombotic pathways in acute coronary syndromes. Am J Cardiol. 2003;91(12A):3H-11H.

8. Angiolillo DJ, Ueno M, Goto S. Basic principles of platelet biology and clinical implications. Circ J. 2010;74(4):597-607.

9. Angiolillo DJ, Fernandez-Ortiz A, Bernardo E, Alfonso F, Macaya $\mathrm{C}$, Bass TA, et al. Variability in individual responsiveness to clopidogrel: clinical implications, management, and future perspectives. J Am Coll Cardiol. 2007;49(14):1505-16.

10. Oh IY, Park KW, Kang SH, Park JJ, Na SH, Kang HJ, et al. Association of cytochrome $\mathrm{P} 4502 \mathrm{C} 19 * 2$ polymorphism with clopidogrel response variability and cardiovascular events in Koreans treated with drugeluting stents. Heart. 2012;98(2):139-44.

11. Man M, Farmen M, Dumaual C, Teng CH, Moser B, Irie S, et al. Genetic variation in metabolizing enzyme and transporter genes: comprehensive assessment in 3 major East Asian subpopulations with comparison to Caucasians and Africans. J Clin Pharmacol. 2010;50(8):929-40.

12. Jinnai T, Horiuchi H, Makiyama T, Tazaki J, Tada T, Akao M, et al. Impact of CYP2C19 polymorphisms on the antiplatelet effect of clopidogrel in an actual clinical setting in Japan. Circ J. 2009;73(8): 1498-503.

13. Jernberg T, Payne CD, Winters KJ, Darstein C, Brandt JT, Jakubowski JA, et al. Prasugrel achieves greater inhibition of platelet aggregation and a lower rate of non-responders compared with clopidogrel in aspirin-treated patients with stable coronary artery disease. Eur Heart J. 2006;27(10):1166-73.

14. Gurbel PA, Bergmeijer TO, Tantry US, et al. The effect of CYP2C19 gene polymorphisms on the pharmacokinetics and pharmacodynamics of prasugrel 5-mg, prasugrel 10-mg and clopidogrel $75-\mathrm{mg}$ in patients with coronary artery disease. Thromb Haemost. 2014;112(3):589-97.

15. Wiviott SD, Braunwald E, McCabe $\mathrm{CH}$, et al. Prasugrel versus clopidogrel in patients with acute coronary syndromes. N Engl J Med. 2007;357(20):2001-15.

16. Mega JL, Close SL, Wiviott SD, Shen L, Hockett RD, Brandt JT, et al. Cytochrome p-450 polymorphisms and response to clopidogrel. N Engl J Med. 2009;360(4):354-62.

17. Mega JL, Close SL, Wiviott SD, Shen L, Hockett RD, Brandt JT, et al. Cytochrome $\mathrm{P} 450$ genetic polymorphisms and the response to prasugrel: relationship to pharmacokinetic, pharmacodynamic, and clinical outcomes. Circulation. 2009;119(19):2553-60.

18. Levine GN, Jeong YH, Goto S, Anderson JL, Huo Y, Mega JL, et al. Expert consensus document: World Heart Federation expert consensus statement on antiplatelet therapy in East Asian patients with ACS or undergoing PCI. Nat Rev Cardiol. 2014;11(10):597-606.

19. Hata J, Kiyohara Y. Epidemiology of stroke and coronary artery disease in Asia. Circ J. 2013;77(8):1923-32.

20. Ueda K, Hasuo Y, Kiyohara Y, Wada J, Kawano H, Kato I, et al. Intracerebral hemorrhage in a Japanese community, Hisayama: incidence, changing pattern during long-term follow-up, and related factors. Stroke. 1988;19(1):48-52.
21. Nakamura M, Iizuka T, Sagawa K, Abe K, Chikada S, Arai M. Prasugrel for Japanese patients with acute coronary syndrome in short-term clinical practice (PRASFIT-Practice I): a postmarketing observational study. Cardiovasc Interv Ther. 2018;33(2):135-45.

22. Thygesen K, Alpert JS, Jaffe AS, Simoons ML, Chaitman BR, White HD. Third universal definition of myocardial infarction. Circulation. 2012;126(16):2020-35.

23. Tunstall-Pedoe H, Kuulasmaa K, Amouyel P, Arveiler D, Rajakangas AM, Pajak A. Myocardial infarction and coronary deaths in the World Health Organization MONICA Project. Registration procedures, event rates, and case-fatality rates in 38 populations from 21 countries in four continents. Circulation. 1994;90(1):583-612.

24. Kimura $\mathrm{K}$, Asai T, Ogawa $\mathrm{H}$ et al. Guidelines for the management of patients with ST-elevation acute myocardial infarction (JCS 2013), article in Japanese, http://www.j-circ.or.jp/guideline/pdf/ JCS2013_kimura_d.pdf

25. Kimura $\mathrm{T}$, Issiki $\mathrm{T}$, Ohno $\mathrm{T}$ et al. Guidelines for management of acute coronary syndrome without persistent ST segment elevation (JCS 2012), article in Japanese, http://www.j-circ.or.jp/guideline/ pdf/JCS2012 kimura d.pdf

26. Wiviott SD, Antman EM, Gibson CM, Montalescot G, Riesmeyer J, Weerakkody G, et al. Evaluation of prasugrel compared with clopidogrel in patients with acute coronary syndromes: design and rationale for the TRial to assess Improvement in Therapeutic Outcomes by optimizing platelet InhibitioN with prasugrel Thrombolysis In Myocardial Infarction 38 (TRITON-TIMI 38). Am Heart J. 2006;152(4):627-35.

27. Cannon CP, Battler A, Brindis RG, et al. American College of Cardiology key data elements and definitions for measuring the clinical management and outcomes of patients with acute coronary syndromes. A report of the American College of Cardiology Task Force on Clinical Data Standards (Acute Coronary Syndromes Writing Committee). J Am Coll Cardiol. 2001;38(7):2114-30.

28. Rao AK, Pratt C, Berke A, Jaffe A, Ockene I, Schreiber TL, et al. Thrombolysis in Myocardial Infarction (TIMI) Trial-phase I: hemorrhagic manifestations and changes in plasma fibrinogen and the fibrinolytic system in patients treated with recombinant tissue plasminogen activator and streptokinase. J Am Coll Cardiol. 1988;11(1):1-11.

29. Mehran R, Rao SV, Bhatt DL, Gibson CM, Caixeta A, Eikelboom $\mathrm{J}$, et al. Standardized bleeding definitions for cardiovascular clinical trials: a consensus report from the Bleeding Academic Research Consortium. Circulation. 2011;123(23):2736-47.

30. Cutlip DE, Windecker S, Mehran R, Boam A, Cohen DJ, van Es $\mathrm{GA}$, et al. Clinical end points in coronary stent trials: a case for standardized definitions. Circulation. 2007;115(17):2344-51.

31. Daida H, Miyauchi K, Ogawa H, Yokoi H, Matsumoto M, Kitakaze $\mathrm{M}$, et al. Management and two-year long-term clinical outcome of acute coronary syndrome in Japan: prevention of atherothrombotic incidents following ischemic coronary attack (PACIFIC) registry. Circ J. 2013;77(4):934-43.

32. Kang J, Han JK, Ahn Y, Chae SC, Kim YJ, Chae IH, et al. Thirdgeneration P2Y12 inhibitors in East Asian acute myocardial infarction patients: a nationwide prospective multicentre study. Thromb Haemost. 2018;118(3):591-600.

33. Kim MC, Jeong MH, Sim DS, Hong YJ, Kim JH, Ahn Y, et al. Comparison of clinical outcomes between ticagrelor and prasugrel in patients with ST-segment elevation myocardial infarctionresults from the Korea Acute Myocardial Infarction RegistryNational Institutes of Health. Circ J. 2018;82:1866-73.

34. Myocardial infarction redefined-a consensus document of the Joint European Society of Cardiology/American College of Cardiology Committee for the redefinition of myocardial infarction. Eur Heart J. 2000;21(18):1502-13. 